\title{
Dengue Fever in Pakistan, Episodes of Epidemic to Endemic: Treatment Challenges, Prevention and Current Facts
}

\author{
Ali $\mathbf{H}^{1^{\star}}$, Alvi $\mathbf{A}^{1}$, Fatima $\mathbf{S}^{1}$, Zafar $\mathrm{F}^{2}$, Naveed $\mathrm{S}^{3}$, Khan $\mathrm{K}^{1}$, Ali $\mathbf{U}^{4}$, Tariq $\mathrm{A}^{5}$, Naqvi GR ${ }^{5}$ and Mallick $\mathbf{N}^{3}$ \\ ${ }^{1}$ Jinnah Sindh Medical University, Karachi, Pakistan \\ ${ }^{2}$ University of Karachi, Karachi, Pakistan \\ 3innah University for Woman Karachi, Karachi 74600, Pakistan \\ ${ }^{4}$ Ayub Teaching Hospital, Abbottabad, Pakistan \\ ${ }^{5}$ Ziauddin University, Karachi, Pakistan
}

\begin{abstract}
Dengue a majorly imperative arthropod-borne infection of viral origin has stroked the humans Worldwide. It has been estimated that about 2.5 billion inhabitants are at threatened by this infection around the globe. Dengue fever has become pandemic-prone viral disease prevailing all over subtropical and tropical regions. Majorly four characteristics of closely related serotypes of dengue are DENV-1, DENV-2, DENV-3 and DENV-4. Dengue fevers pass on by the bite of an infected female Aedes aegypti mosquito. The topmost ailments include; internal bleeding, Dengue Hemorrhagic Fever (DHF) which may lead to Circulatory Shock Syndrome (CSS), high temperature, cold like illness, urticaria, severe joint pain, thrombocytopenia and heme-concentration. From the recent years, this "breakbone fever" has become out breaking viral disease in Pakistan because Urbanization provided the ideal environment for these mosquitoes which includes presence of stagnant water in indoor drainage holes, contaminated water for drinking, poverty, immune compromised individuals and insufficient medical facilities. Such causative factors embrace viral disease that brings about high rate of mortality and morbidity. But nowadays, rational prescribing and the facilities provided by pharmaceutical care has become necessary element of health care for achieving definite outcomes that can improve a patient's quality of life.
\end{abstract}

Keywords: Dengue Hemorrhagic Fever (DHF); Circulatory Shock Syndrome (CSS); Viral disease; Pharmaceutical care; Mortality

\section{Introduction}

Dengue was first discovered in $20^{\text {th }}$ century as one of the encyclopedic mosquito borne infection in human beings. The dengue virus, as an arthropode-borne virus belongs to genus Flavivirus and family Flaviviridae. As per The World Health Organization (WHO), dengue infection is now endemic in some parts of Asia and approximates that globally 50 million individuals infected by dengue every year, out of which the mortality rate is 24,000 [1-3]. The synonym for dengue is "break bone fever" i.e., the severe joint pain. The dengue is infecting 30 times more, from 1960 to the year 2010 [4,5]. Centers for Disease Control and Prevention (CDC) and WHO states that, In Pakistan and in other Asian countries dengue fever is a major health problem $[6,7]$.

\section{Epidemiology and Pervasiveness}

In Pakistan, during the post monsoon period, the highest cases of far-flung mosquito borne disease (dengue fever) has been narrated especially after the incidence of floods resulting in transform of disease status from endemic to epidemic [6]. In Pakistan, during the year of 1994, first epidemic dengue has been exposed [3].

High prevalence of dengue was reported in Pakistan in the post monsoon season. Nonetheless the problem has been speeded peacefully and turns this scenario of dengue fever from endemic to pandemic. Situation becomes more worst after the major flood strikes in the country. The most prevalent virus was noted to be DENV-2 (96\%) than other serotypes [3,7]. In 2003, ten cases with 4 deaths, while an approximate of 14000 incidences were reported in 2011 with death rate of $2.14 \%(\mathrm{~N}=300)$. A massive threat has been noted statistically between 2005-2010. Till 2010, the cases of dengue had come to light as an endless hazard for inhabitants reporting increased in incidents from 4500 to 21204. In view of the fact that dengue incidence was potentially pitched up to over four thousand cases annually and this larger and horrific estimate in recent past including 365 deaths with 21597 affirmative dengue cases convert this hazardous situation endemic to Pakistan [7-9]. An array in this estimate may be associated with poor reporting system [10-12].

\section{Serotypes}

The four distinct viruses (DENV-1 to DENV-4) of dengue but possess the same geographic and environmental locus. These four viruses are called serotypes because each has different interactions with the antibodies in human blood serum. Out of these 4 serotypes, the most life-threatening serotype is DENV-2, whereas Dengue Hemorrhagic Fever (DHF) is concomitant with DENV-2 and DENV-3 but still the underlying cause is unspecified [4-8].

\section{Etiology}

The viruses which belong to the Flaviviridae family are the main causative agents of dengue fever and other infectious diseases. The augmentation of contagion depends upon the spread of virus. There are 2 principle vectors of dengue i.e., Aedes aegypti (most prevalent) and Aedes albopictus (the most invasive and protruding mosquito worldwide) [9]. The incubation period of dengue fever is 4-7 days.

*Corresponding author: Ali H, Faculty of Pharmacy, Jinnah Sindh Medical University, Karachi, Pakistan, Tel: +0092-03002621917; E-mail: humaali80@live.com

Received April 22, 2017; Accepted June 12, 2017; Published July 28, 2017

Citation: Ali H, Alvi A, Fatima S, Zafar F, Naveed S, et al. (2017) Dengue Fever in Pakistan, Episodes of Epidemic to Endemic: Treatment Challenges, Prevention and Current Facts. J Bioequiv Availab 9: 473-476. doi: 10.4172/jbb.1000347

Copyright: () 2017 Ali H, et al. This is an open-access article distributed under the terms of the Creative Commons Attribution License, which permits unrestricted use, distribution, and reproduction in any medium, provided the original author and source are credited. 
Non-specific type of symptoms is: febrile, anxiety, rash and respiratory tract infections while high grade fever, annoyance, body pain and maculopapular rash are the typical symptoms [10]. The distinctive characters of Dengue hemorrhagic fever (DHF) and dengue shock syndrome (DSS) based on internal bleeding which can lead to comorbid conditions [13-17].

\section{Major Reasons of Disease Propagation in Pakistan}

The key factors that influencing the disease propagation in Pakistan are; increased residents rate, poor sanitation, inflation in population, unhygienic food and water, inadequate medical and pharmaceutical care facilities, co-morbidity, high illiteracy rate, less knowledge regarding vaccination, less awareness about preventive measures taken to prevent disease $[9,12-13]$. Even though surveillance and official coverage of dengue to WHO from African and Eastern Mediterranean constituencies are poor, but in 2005-2006. The epidemics of alleged dengue were documented in Pakistan, Yemen, Saudi Arabia, Madagascar and Sudan [18-20].

\section{Symptoms and Clinical Consequences}

All over the world, the underlying principle behind the amplification of dengue virus is interaction of all 4 serotypes of dengue virus with antibodies and human blood serum. Patients bearing dengue will have a history of living in or recent travel to, a region where the disease is endemic [1]. A wide spectrum of illness can be observed, extending from barely visible, mild disease, called dengue fever, to a severe and sporadically fatal dengue hemorrhagic fever/dengue shock syndrome [8,21]. In March 2010, the most persuasive factor that causing the deaths of patient with dengue fever are disturbed neurological signs (The neuropathogenesis of DENV infection is still poorly understood), impaired renal functions and bleeding. The symptoms of mild dengue fever are: arthralgia, body rash, high fever, intense headache and nauseatic feeling. Mild to severe clinical manifestations are: Dengue Hemorrhagic Fever (DHF) and if left untreated, DHF may progress to dengue shock syndrome (DSS) [8]. As per the research held in Singapore, mortality rate is going on, due to the co-morbid conditions that develop along with dengue fever. The clinical symptoms that present during the recovery phase are based on an individual's immunity [22-26].

\section{Diagnosis}

Within the first few bouts of fever in patients recognized with dengue virus, it is hard to distinguish whether it is dengue fever or dengue hemorrhagic fever therefore; both conditions are differentiated by viral hemorrhagic fever like measles, influenza, typhoid, malaria, etc. According to WHO (World Health Organization), the benchmark of hemorrhagic fever [27-29] is for patient to exhibit fever as well as hemorrhaging features, thrombocytopenia along with vascular permeability [30,31].

\section{Treatment Considerations}

Early and symptomatic treatment is required for fever in patients. Fluid restoration orally must be provided or intravenous administration of $0.9 \%$ saline, ringer's lactate, Hartmann's solution. Rest must be advised necessarily. With respect to clinical considerations (age, temperature, etc.), Acetaminophen (paracetamol) should be given either orally or intravenously (IV). Initiation of NSAID (Non-steroidal Anti-inflammatory Drugs) may lead to exacerbation of bleeding in patients and are therefore contraindicated [32-34].

Various studies disapproved antibiotic therapy in patient with dengue fever along with one's with severe neutropenia. Nevertheless, in patients with potential bacteremia, antibiotic empiric therapy can be given [35]. Studies also suggest use of RNA interference against viruses of family Flaviviridae, to suppress viral genome. It not only works against dengue virus but also has activity against Poliovirus, Influenza $\mathrm{A}$ virus, Human Immunodeficiency virus (HIV) type-1, Hepatitis $C$ virus (HCV), Hepatitis B virus (HBV) [36].

\section{Control and Vaccination}

As in many other diseases, ways to restrain pathogens are very beneficial in eliminating disease and so the dengue virus population is infected through the bacteria from obligate endosymbiont, genus Wolbachia ( $W$. pipients). Transformation and transmission sequence in pathogens are disturbed and resistance is produced. It is a gramnegative bacterium that disturbs the host reproduction mechanism. Its Anti-viral effects are produced by shortening the lifespan of virus inside mosquito thus, exploiting the viral gene expression of host. Pathogens are also eliminated through herbal agents as well as anti-viral amalgamations are in use against dengue virus. New modified anti-viral agents (against all four serotypes) that are safe, inexpensive but effective at the same time are, however, a growing need $[9,37,38]$.

The biomedical technological area has development of vaccine for dengue in progress but the fact that dengue virus has four robust serotypes is an opposition in its way. It would be an irreplaceable and immense benefit to public health to attain a vaccine that is live tetravalent, safe and effective against dengue virus [39]. A study in region prevalent to dengue fever showed that vaccine had immunogenicity and proved to be safe in majority of patient's event when somewhere predisposed to the virus. Most patients from age 1.5-45 years were responsive to TDV (Tetravalent Dengue Vaccine). In Philippines, first ever dengue vaccine, Dengvaxia, after several prolonged trials, has been approved and allowed to be used in Asia [40,41]. Dengue is one of the most prevalent mosquitos borne disease throughout the World according to WHO therefore, Sanofi Pasteur designed this vaccine. Even though this vaccine doesn't have sufficient evidence to prove its tolerance in children younger than 9 years but the risk-benefit ratio suggests it to be advantageous for adult cases. However, to be on a safer side, the Philippines department of Health has arranged immunization campaigns in most dengue receptive areas $[42,43]$.

\section{Economic Impact}

Dengue lays significant effects on the economy and social status along with its drastic consequences on public health. From 2005-2006, as a study shows, it cost about $\$ 829[32,44]$. Also, high social destruction causes unsuccessful health care facilities to sufferers [45-47].

In a cross-sectional investigation carried out in different cities of Pakistan during 2012 to 2013. Socio-economic status of most suffered population was calculated along with direct and indirect costs of treatment. 58, 28 and $14 \%$ of study respondents belong to low, middle and high socioeconomic cohort respectively. In particular the disability life years adjusted per million populations was noted to be 133.76 [48]. Furthermore, in another study it has been reported that Disabilityadjusted Life Years (DALYs) per million populations in endemic regions of Asia is more or less 1,300 which is in an equal agreement to the infection encumbered by the tropical and childhood ailments, like tuberculosis, in such regions [49].

\section{Preventive Strategies}

Apart from traditional preventive measures, pesticides (although 
few species are resistant to pesticide use) are used to remove larval mosquito habitat and community awareness campaigns are organized. More so, new researches are demand of time to study at the exact points of location of dengue virus transmission and promotion $[14,50]$.

\section{Management of Dengue Infection}

During febrile illness, all patients must be provided with supportive as well symptomatic treatment. Acetaminophen should be initiated either oral or intravenous. Administration of intravenous fluids such as $0.9 \%$ saline, Ringer's lactate, $5 \%$ glucose diluted $1: 2$ or $1: 1$ in normal saline, Hartmann's solution. Non-steroidal anti-inflammatory agents must not be initiated owing to their anti-platelet effects that can lead to increased bleeding tendencies in patients. Administration of volume expanders and crystalloids. Administration of dengue vaccine.

As per WHO guidelines, the principles of fluid therapy administration [50]:

- In severe cases like circulatory shock, unconscious patient, and in severe vomiting it is mandatory to administered intravenous fluid.

- Plenty of oral fluid should be taken.

- First preference for intravenous fluid (0.9\% saline) are Ringer solution (crystalloids).

- Dextran (colloids) form the second line measures for the hypotensive state patients that do not respond to crystalloids (intravenous).

- In the critical phase, when the patient is severely bleeding and platelet counts are very low than the fresh whole blood transfusion is the best way of management.

\section{Recent Investigation to Combat Disease Burden}

Aims to prevent and treat dengue infection are to control vector transmission, development of dengue vaccine, and antiviral drugs. Vector transmission can be controlled by keeping Poecilia reticulate (guppies) or Doridicola agilis (copepods) in stagnant water, which infects the mosquitoes with bacteria of the Wolbachia genus [51]. The investigators persuaded the spread and pattern of Wolbachia using mosquito populations around two years by ensnaring and studying them for the specific bacteria [52]. Considerations should be made on the development of dengue vaccine due to the enhancing severity of dengue infection and increased transmission. Major obstacles in the development and progress of dengue vaccines are mainly associated with complex serology of viral origin, multifaceted pathology and inadequate investment by vaccine designers [53]. Careful attempts are aimed in the advancement of antiviral drugs that can shun life threatening episodes regarding dengue fever [54]. Most recently a research group studied the covalently linked dengue virus envelope glycoprotein dimers in order to decrease the revelation of the immunedominant combination loop epitope. Such locked dimers are strapping immunogen contenders for next-generation producers of vaccine [55].

\section{Conclusion}

The damage caused by dengue virus can be reduced through preventive measures against pathogen and empiric treatment for dengue fever and so the illness goes away with time. Studies are demanded to investigate different features of mosquito and dengue viruses that might further prove to be helpful in finding new efficacious techniques for betterment of health care system. Major areas of concern are control of vectors, management of disease and new community awareness programs. Spontaneous educational supply, workshops, training of personals, related literature provision for control, prevention and management of the disease are required by the health care professionals. Also, the electronic media can perform a significant role in spreading awareness in people. The transmission can be limited through various preventive strategies such as spraying, fumigation, repellents and water coverings. Therefore, to eradicate dengue from Pakistan, a combined effort from several organizations, health teams, educational programs and disambiguation means are required. Rational therapy, pharmaceutical and clinical support is needed for patient welfare.

\section{References}

1. Jahan F (2011) Dengue Fever (DF) in Pakistan. Asia Pac Fam Med 10: 1.

2. The World Health Organization (WHO) (2009) Dengue and severe dengue. Dengue Fever World Health Organization Fact Sheet No. 1172009.

3. Ahmad T (2013) Dengue virus serotype 2 (DEN-2) is common in Pakistan. Am J Biomed Sci 5: 250-252.

4. Sadia N, Muhammad A, Ashraf M, Ahmad R (2015) The epidemiology of dengue fever in district Faisalabad, Pakistan. IJSRP 5: 1-6.

5. Ahmed S, Ali N, Ashraf S, Ilyas M, Tariq WU, et al. (2008) Dengue fever outbreak: a clinical management experience. J Coll Physicians Surg Pak 18: 8-12.

6. Idrees S, Ashfaq UA (2012) A brief review on dengue molecular virology, diagnosis, treatment and prevalence in Pakistan. Genet Vaccines Ther 10: 6.

7. Ali J (2015) Dengue fever in Pakistan: Challenges, priorities and measures. $J$ Coast Life Med 3: 834-837.

8. Almas A, Parkash O, Akhter J (2012) Clinical factors associated with mortality in dengue infection at a tertiary care center. Southeast Asian J Trop Med Public Health 41: 333-340.

9. Tahir U, Khan UH, Zubair MS (2015) Wolbachia pipientis: A potential candidate for combating and eradicating dengue epidemics in Pakistan. Asian Pac J Trop Dis 8: 989-998.

10. Rafiq MK, Arif A, Shaikh R (2011) Dengue in Pakistan: Journey from a disease free to a hyper endemic nation. Editorial Board 5: 81.

11. Shahid A, Wasim WM, Faran H, Amim A, Raja K, et al. (2013) the 2011 dengue hemorrhagic fever outbreak in Lahore-an account of clinical parameters and pattern of hemorrhagic complications, J Coll Physicians Surg Pak 23: 463-467.

12. Zulfiqar AB, Johannes S, Zohra SL, Rehana AS, Jai KD (2014) Global burden, distribution, and interventions for infectious diseases of poverty. Infect Dis Poverty 3:21.

13. Khalid K, Ghufran U, Mike BT (2012) The Emergence and Maintenance of vector-borne diseases in the Khyber Pakhtunkhwa province, and the federally administered tribal areas of Pakistan. Front Physiol 3: 250.

14. Khan E, Hasan R (2011) Dengue infection in Asia: A regional concern. J Postgrad Med Inst 26: 01-06.

15. Benedict MQ, Levine RS, Hawley WA, Lounibos LP (2007) Spread of the tiger: global risk of invasion by the mosquito Aedes albopictus vector borne. Zoonotic Dis Spring 7: 76-85.

16. Khan E, Hasan R, Mehraj V, Nasir A, Siddiqui J, et al. (2008) Co-circulations of two genotypes of dengue virus in 2006 out-break of dengue hemorrhagic fever in Karachi, Pakistan J Clin Virol 43: 176-179.

17. World Health Organization (WHO) (2007) Scientific working group report on dengue. Geneva, Switzerland: World Health Organization.

18. Guzman MG, Kouri G (2002) Dengue: an update. Lancet Infect Dis 2: 33-42.

19. Gubler DJ (2004) The changing epidemiology of yellow fever and dengue, 1900 to 2003: full circle? Comp Immunol Microbiol Infect Dis 27: 319-330.

20. Muhammad A, Nadeem S, Ibrar R, Saira B, Arsalan AS (2014) Retrospective 
Citation: Ali H, Alvi A, Fatima S, Zafar F, Naveed S, et al. (2017) Dengue Fever in Pakistan, Episodes of Epidemic to Endemic: Treatment Challenges, Prevention and Current Facts. J Bioequiv Availab 9: 473-476. doi: 10.4172/jbb.1000347

analysis of dengue fever case management and frequency of co-morbidities associated with deaths. BMC Res Notes 7: 205.

21. Bhattacharya MK, Maitra S, Ganguly A, Bhattacharya A, Sinha A (2013) Dengue: A growing menace-a snapshot of recent facts, figures \& remedies. Int J Biomed Sci 9: 61-67.

22. Westaway EG, Blok J (1997) Taxonomy and evolutionary relationships of flaviviruses. Dengue and dengue hemorrhagic fever 147-173.

23. Hirsch A (2000) Dengue, a comparatively new disease: its symptomsgeographic distribution-characteristics of dengue as an epidemic disease of the tropics. Handbook of Geographical and Historical Pathology 1: 466-520.

24. Hayes EB, Gubler DJ (1992) Dengue and dengue hemorrhagic fever. Pediatr Infect Dis J 11: 311-317.

25. Low JG, Ooi EE (2013) Dengue-old disease, new challenges in an ageing population. Ann Acad Med Singapore 42: 373-375.

26. Gubler DJ (1998) Dengue and dengue hemorrhagic fever. Clin Microbiol Rev 11: $480-496$.

27. Thai KT, Nishiura H, Hoang PL, Tran NTT, Phan GT, et al. (2011) Agespecificity of clinical dengue during primary and secondary infections. PLOS Negl Trop Dis 5: e1180.

28. Peeling RW, Artsob H, Pelegrino JL, Buchy P, Cardosa MJ, et al. (2010) Evaluation of diagnostic tests: dengue. Nat Rev Microbiol 8: 30-37.

29. Rigau PJG (1997) Clinical manifestations of dengue hemorrhagic fever in Puerto Rico. Puerto Rico Assoc Epidemiol 1: 381-388.

30. Siler JF, Hall MW, Hitchens A (1996) Dengue, its history, epidemiology, mechanism of transmission, etiology, clinical manifestations, immunity and prevention. Philipp J Sci 29: 1-304.

31. World Health Organization (2009) Special Programme for Research, Training in Tropical Diseases. Department of Control of Neglected Tropical Diseases, World Health Organization. Epidemic, \& Pandemic Alert. Dengue: guidelines for diagnosis, treatment, prevention and control. World Health Organization.

32. Barrera R, Delgado N, Jiménez M, Valero, S (2002) Eco-epidemiological factors associated with hyperendemic dengue haemorrhagic fever in Maracay City, Venezuela.

33. Thein TL, Lye DC, Leo YS, Wong JG, Hao Y, et al. (2014) Severe neutropenia in dengue patients: prevalence and significance. Am J Trop Med Hyg 90: 984-987.

34. Sobia I, Usman AA (2013) RNAi: antiviral therapy against dengue virus. Asian Pac J Trop Biomed 3: 232-236.

35. Hussain M, Frentiu FD, Moreira LA, O'Neill SL, Asgari S (2011) Wolbachia uses host micro RNAs to manipulate host gene expression and facilitate colonization of the dengue vector Aedes aegypti. Proc Natl Acad Sci 108: 9250-9255.

36. Hoang LT, Lynn DJ, Henn M, Birren BW, Lennon NJ, et al. (2010) The early whole-blood transcriptional signature of dengue virus and features associated with progression to dengue shock syndrome in Vietnamese children and young adults. J Virol 84: 12982-12994.

37. Sirivichayakul C, Barranco-Santana EA, Stinchcomb DT (2013) Safety and immunogenicity of a tetravalent dengue vaccine (TDV) in healthy children and adults in endemic regions: a randomized, placebo-controlled Phase 2 study. Vaccine 31: 5814-5821.

38. Hadinegoro SR, Arredondo-García JL, Capeding MR, Deseda C, Chotpitayasunondh T, et al. (2015) Efficacy and long-term safety of a dengue vaccine in regions of endemic disease. N Engl J Med 373: 1195-1206.

39. Thomas SJ, Endy TP (2011) Vaccines for the prevention of dengue: development update Hum. Vaccin 7: 674-684.

40. Cameron PS (2015) A candidate dengue vaccine walks a Tightrope. N Engl J Med 373: 1263-1264.

41. Nagao Y, Tawatsin A, Thammapalo S, Thavara U (2012) Geographical gradient of mean age of dengue haemorrhagic fever patients in northern Thailand. Epidemiol Infect 140: 479-490.
42. Gubler DJ (2002) Epidemic dengue/dengue hemorrhagic fever as a public health, social and economic problem in the $21^{\text {st }}$ century. Trends Microbiol 10 : 100-103.

43. Duane JG (2012) The economic burden of Dengue. Am J Trop Med Hyg 86 743-744.

44. Ibrar R, Arif MNS, Muhammad AM, Shajee S, Huma Q, et al (2015) Economic burden of dengue in four major cities of Pakistan during 2011. J Pak Med Assoc 65: 256-259.

45. Gubler DJ, Meltzer M (1999) Impact of dengue/dengue hemorrhagic fever on the developing world. Adv Virus Res 53: 35-70.

46. Reiter P, Goh KT (1998) Dengue control in Singapore. In: Dengue in Singapore. Singapore: Institute of Environmental Epidemiology. Ministry of the Environment 213-238.

47. Eng-Eong O, Kee TG, Duane JG (2006) Dengue prevention and 35 years of vector control in Singapore. Emerg Infect Dis 12: 887-893.

48. World Health Organization (2009) Dengue: Guidelines for diagnosis, treatment prevention and control.

49. Simmons CP, Farrar JJ, Nguyen V, Wills B (2012) Dengue. N Engl J Med 366 1423-1432.

50. Schmidt TL, Barton NH, Rašić G, Turley AP, Montgomery BL, et al. (2017) Local introduction and heterogeneous spatial spread of dengue-suppressing Wolbachia through an urban population of Aedes aegypti. PLoS Bio 15 e2001894.

51. Hombach $\mathrm{J}$ (2007) Vaccines against dengue: A review of current candidate vaccines at advanced development stages. Rev Panam Salud Pública 21 254-260.

52. Noble CG, Chen YL, Dong H, Gu F, Lim SP, et al. (2010) Strategies for development of dengue virus inhibitors. Antiviral Res 85: 450-462.

53. Sampath A, Padmanabhan R (2009) Molecular targets for flavivirus drug discovery. Antiviral Res 81: 6-15.

54. Hasan S, Jamdar SF, Alalowi M, Al-Beaiji SMAA (2016) Dengue virus: A global human threat: Review of literature. J Int Soc Prev Commun Dent 6: 1.

55. Rouvinski A, Dejnirattisai W, Guardado-Calvo P, Vaney MC, Sharma A, et al. (2017) Covalently linked dengue virus envelope glycoprotein dimers reduce exposure of the immunodominant fusion loop epitope. Nat Commun 8. 